Case Report

\title{
Hyperkalemia Induced by the Sequential Administration of Metoprolol and Carvedilol
}

\author{
S. Serge Barold $\mathbb{D D}^{1}$ and Scott Upton ${ }^{2}$ \\ ${ }^{1}$ Department of Medicine, University of Rochester School of Medicine and Dentistry, Rochester, NY, USA \\ ${ }^{2}$ San Diego Internal Medicine and Pediatrics Associates, San Diego, CA, USA \\ Correspondence should be addressed to S. Serge Barold; ssbarold@aol.com
}

Received 23 July 2018; Accepted 24 September 2018; Published 15 October 2018

Academic Editor: Magnus Baumhäkel

Copyright (c) 2018 S. Serge Barold and Scott Upton. This is an open access article distributed under the Creative Commons Attribution License, which permits unrestricted use, distribution, and reproduction in any medium, provided the original work is properly cited.

\begin{abstract}
This report describes the occurrence of asymptomatic hyperkalemia induced by the sequential administration of metoprolol and carvedilol in an 81-year-old man with type II diabetes and stable stage III renal insufficiency. The potassium level rose to $5.6-5.7 \mathrm{mEq} / \mathrm{L}$ with metoprolol and normalized when the agent was discontinued. However, the potassium level rose again to $5.6 \mathrm{mEq} / \mathrm{L}$ after the administration of carvedilol but the level normalized by halving the dose. The observations of hyperkalemia induced by two different $\beta$-blocker drugs in the same patient confirm that this side effect is common to all $\beta$-blocker drugs.
\end{abstract}

\section{Introduction}

Hyperkalemia is a rare side effect of $\beta$-blocker drugs [1-3]. In this report, we describe the occurrence of metoprololinduced hyperkalemia in a patient with type 2 diabetes and stage III renal dysfunction. The potassium level normalized after metoprolol was discontinued, but the administration of carvedilol subsequently induced hyperkalemia. The development of hyperkalemia in the same patient induced by two different $\beta$-blocker drugs has not been previously reported.

\section{Case Report}

An 81-year-old man presented with asymptomatic of hyperkalemia $(5.7 \mathrm{mEq} / \mathrm{L})$. The past history included mitral valve repair and coronary bypass surgery 27 years previously, mild type II diabetes for 12 years, renal insufficiency (stage III) with a stable creatinine level of $1.5 \mathrm{mg} / \mathrm{dL}$ for 8 years and prostatic hypertrophy. An echocardiogram performed 6 months previously revealed normal left ventricular function and minimal mitral regurgitation and an ECG documented sinus rhythm and complete left bundle branch block. Medications included Avodart $0.5 \mathrm{mg} \mathrm{qd}$, atorvastatin $80 \mathrm{mg} \mathrm{qd}$, aspirin $325 \mathrm{mg}$ qd, Januvia $25 \mathrm{mg}$ $\mathrm{qd}$, and metoprolol tartrate $50 \mathrm{mg}$ bid. The latter had been prescribed 6 years previously for frequent symptomatic atrial premature beats with an excellent clinical result so that metoprolol at the same dose (all tartrate preparation) was continued up to the most recent evaluation. The potassium level had always been normal before the administration of metoprolol. Afterwards, routine testing with serum electrolytes every 6 or 12 months consistently revealed a potassium level of $5.4 \mathrm{mEq} / \mathrm{L}$. Then, for the last 2 years regular routine testing revealed a potassium level fluctuating between 5.6 and $5.7 \mathrm{mEq} / \mathrm{L}$. Finally, metoprolol was discontinued and 18 days later the potassium level normalized at $4.2 \mathrm{mEq} / \mathrm{L}$. Carvedilol was started and then increased to $12.5 \mathrm{mg}$ bid. After about 10 days on this dose, the potassium level increased to $5.6 \mathrm{mEq} / \mathrm{L}$. The dose of carvedilol was therefore reduced to $6.25 \mathrm{mg}$ bid. A follow-up potassium level 2 weeks after the start of the lower carvedilol dose was $5.0 \mathrm{mEq} / \mathrm{L}$ which is at the upper limit of normal for the testing laboratory. 


\section{Discussion}

$\beta$-Blocker-induced hyperkalemia is a rare nonspecific side effect of $\beta$-blocker therapy as illustrated by the effect from two different $\beta$-blockers in the same patient. It appears from a review of the literature that a biological difference between the 2 available forms of metoprolol, tartrate or succinate, is unlikely.

3.1. Metoprolol. A 2018 FDA report evaluated the incidence of hyperkalemia (level not stated) in 24,296 patients taking metoprolol succinate and found 287 patients with hyperkalemia [4]. The patients with hyperkalemia were taking ramipil $(41.5 \%)$ or spironolactone $(10.4 \%)$. No other potassiumretaining drugs were taken. An increased creatinine was present in $20.56 \%$ and acute renal failure in $33.80 \%$ of the patients. The FDA report suggests that metoprolol-induced hyperkalemia occurs in about $0.5 \%$ of the patients without acute renal failure and can occur in patients without diabetes or renal dysfunction. Yet, there are only two Medline citations about this side effect of metoprolol. One case involved a 45-year-old hypertensive diabetic man with advanced renal failure on hemodialysis who developed marked recurrent hyperkalemia while on metoprolol therapy (full preparation not stated) [1]. The other case documented hyperkalemia as a result of a suicide with metoprolol [5].

3.2. Propranolol. Propranolol has rarely been reported to induce hyperkalemia in the adult patient, though it is well documented as a serious problem when it is used to treat hemangiomas in infants $[3,6,7]$.

3.3. Carvedilol. In our case, the potassium level rose to $5.6 \mathrm{mEq} / \mathrm{L}$ when the patient (with type III renal insufficiency) was on carvedilol $12.5 \mathrm{mg}$ bid but it normalized (though at the upper limit of normal $-5 \mathrm{mEq} / \mathrm{L}$ ) when the dose of carvedilol was reduced to $6.25 \mathrm{mg}$ bid. A similar observation was recently reported about carvedilol-induced hyperkalemia in a 69-year-old man with hypertension, type II diabetes, and stage III renal insufficiency who was hospitalized for abdominal pain [2]. He had been on carvedilol $3.125 \mathrm{mg}$ bid and lisinopril $40 \mathrm{mg}$ qd. The serum potassium was $4.8 \mathrm{mEq} / \mathrm{L}$. When carvedilol was increased to $6.25 \mathrm{mg}$ bid, the serum potassium rose to $6.7 \mathrm{mEq} / \mathrm{L}$ without any change in lisinopril dosage or administration of other potassium-retaining drugs. A simple reduction back to the original dose normalized the potassium level [2]. These two reports suggest that the development of hyperkalemia is dose dependent.

3.4. Labetalol. Intravenous labetalol has been associated with severe hyperkalemia for the treatment of acute hypertension in hemodialysis patients, renal transplant patients, and in preeclampsia [8-10].

3.5. Timolol Eyedrops. Severe hyperkalemia occurred in a patient with glaucoma following the use of timolol maleate eyedrops, but the serum potassium level normalized upon discontinuation of the eyedrops [11]. However, hyperkalemia recurred following a rechallenge with the same eyedrops and the potassium level normalized again when the eyedrops were withdrawn.

\section{Mechanisms}

It is well known that adrenergic agents decrease serum potassium and hence are used to treat life-threatening hyperkalemia. $\beta 2$ adrenergic agonists drive potassium into the cells by increasing the activity of the Na-K pump. Thus, a catecholamine surge tends to lower the serum potassium [12]. They also activate the inwardly directed $\mathrm{Na}-\mathrm{K}-\mathrm{Cl}$ cotransporter, a protein that aids in the active transport of sodium, potassium, and chloride into cells [12]. Yet, the opposite effect with the production of hyperkalemia by the inhibition of the sympathetic system as with $\beta$-blockers has only occasionally been reported. The propensity to hyperkalemia may be masked or unrecognized because $\beta$-blockers are commonly administered together with drugs that affect potassium balance.

Barring the acute administration of a $\beta$-blocker drug, there are only 4 well-documented case reports of $\beta$-blockerinduced hyperkalemia from sustained therapy ( 3 from the literature and one described herein). Three cases had associated renal dysfunction and diabetes, while one had none of these associations. Insulin similar to epinephrine enhances the activity of the Na-K pump so that an anti-insulin effect in diabetes would favor the movement of potassium outside the cells [12]. Renal insufficiency decreases the cell membrane potential and thus leads to translocation of potassium to the extracellular fluid [13]. The above clinical cases confirm the FDA data that renal insufficiency is a strong predisposing factor to the development of $\beta$-blocker-induced hyperkalemia. These cases also suggest a probable association with diabetes, though the FDA data revealed a $10 \%$ incidence perhaps because of a different patient population. Finally, it is possible that hyperkalemia may be due to a genetic predisposition.

\section{Conclusion}

We have reported metoprolol-induced and carvedilolinduced hyperkalemia. Such hyperkalemia is rare and seems to be a common side effect of $\beta$-blockers. Hyperkalemia is more common in patients with renal insufficiency and probably in those with diabetes. Identification of this form of hyperkalemia may avoid unnecessary investigations and prevent untoward effects. In patients with stable renal insufficiency, $\beta$-blocker-induced hyperkalemia should not be attributed to progression of kidney disease causing unwarranted alarm and inappropriate therapy. This form of hyperkalemia may be dose dependent so that normalization of the potassium level may not necessarily require complete withdrawal of therapy.

\section{Conflicts of Interest}

The authors declare that they have no conflicts of interest. 


\section{References}

[1] O. S. Ashouri, "Metoprolol-induced hyperkalemia in a diabetic with advanced renal failure," Archives of Internal Medicine, vol. 145, no. 3, p. 578, 1985.

[2] L. Hahn and M. Hahn, "Carvedilol-induced hyperkalemia in a patient with chronic kidney disease," Journal of Pharmacy Practice, vol. 28, no. 1, pp. 107-111, 2015.

[3] D. Mandić, L. Nezić, and R. Skrbić, "Severe hyperkalemia induced by propranolol," Medicinski Pregled, vol. 67, no. 5-6, pp. 181-184, 2014.

[4] "Toprol and hyperkalemia," From an FDA report May 2018, https://www.ehealthme.com/ds/metoprolol-succinate/ hyperkalemia/.

[5] J. L. Delacour, P. L. Blanc, G. Wagschal, and P. Daoudal, "Hyperkalemie au cours d'une intoxication aigue par betablocqueur," Presse Médicale, vol. 15, p. 1377, 1988.

[6] B. Belen, A. Oguz, A. Okur, and B. Dalgic, "A complication to be aware of: hyperkalaemia following propranolol therapy for an infant with intestinal haemangiomatozis," BMJ Case Reports, vol. 2014, 2014.

[7] H. Pavlakovic, S. Kietz, P. Lauerer, M. Zutt, and M. Lakomek, "Hyperkalemia complicating propranolol treatment of an infantile hemangioma," Pediatrics, vol. 126, no. 6, pp. e1589e1593, 2010.

[8] B. Thomas, P. V. Abdul Rouf, W. E. Kassem et al., "A case of probable labetalol induced hyperkalaemia in pre-eclampsia," International Journal of Clinical Pharmacy, vol. 36, no. 6, pp. 1130-1133, 2014.

[9] A. Hamad, M. Salameh, M. Zihlif, D. A. Feinfeld, and C. P. Carvounis, "Life-threatening hyperkalemia after intravenous labetolol injection for hypertensive emergency in a hemodialysis patient," American Journal of Nephrology, vol. 21, no. 3, pp. 241-244, 2001.

[10] S. Arthur and A. Greenberg, "Hyperkalemia associated with intravenous labetalol therapy for acute hypertension in renal transplant recipients," Clinical Nephrology, vol. 33, no. 6, pp. 269-271, 1990.

[11] E. R. Swenson, "Severe hyperkalemia as a complication of timolol, a topically applied $\beta$-adrenergic antagonist," Archives of Internal Medicine, vol. 146, no. 6, pp. 1220-1221, 1986.

[12] D. B. Mount, "Disorders of potassium balance," in Brenner and Rector's The Kidney, B. M. Brenner, Ed., pp. 559-600, WB Saunders Co, Philadelphia, PA, USA, 10th edition, 2016.

[13] J. R. Cotton, T. Woodard, N. W. Carter, and J. P. Knochel, "Resting skeletal muscle membrane potential as an index of uremic toxicity: a proposed new method to assess adequacy of hemodialysis," The Journal of Clinical Investigation, vol. 63, no. 3, pp. 501-506, 1979. 


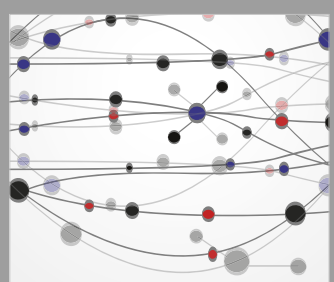

The Scientific World Journal
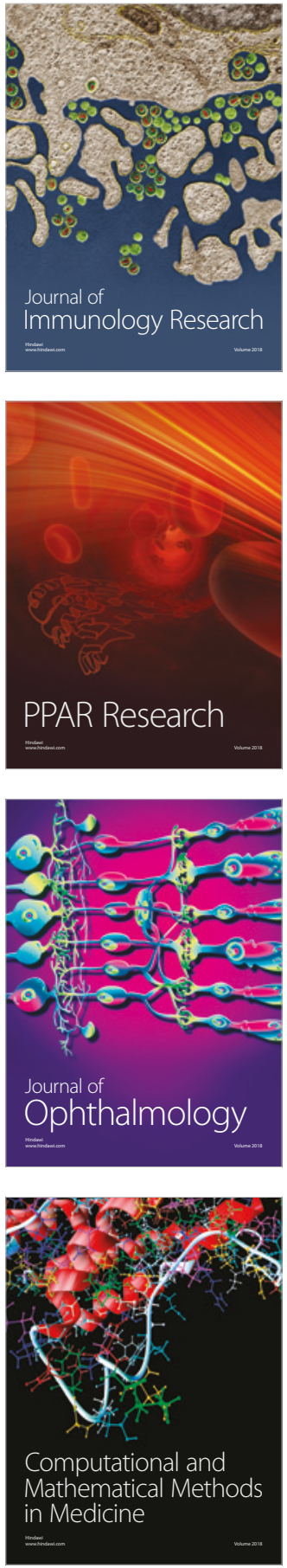

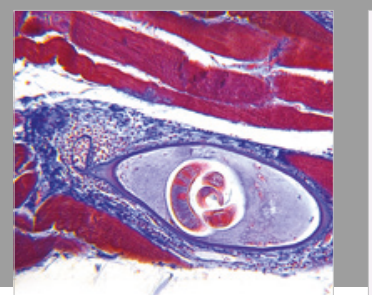

Gastroenterology Research and Practice

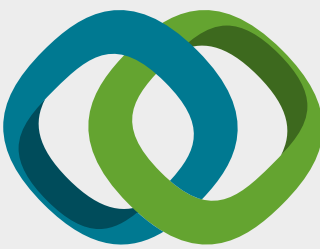

\section{Hindawi}

Submit your manuscripts at

www.hindawi.com
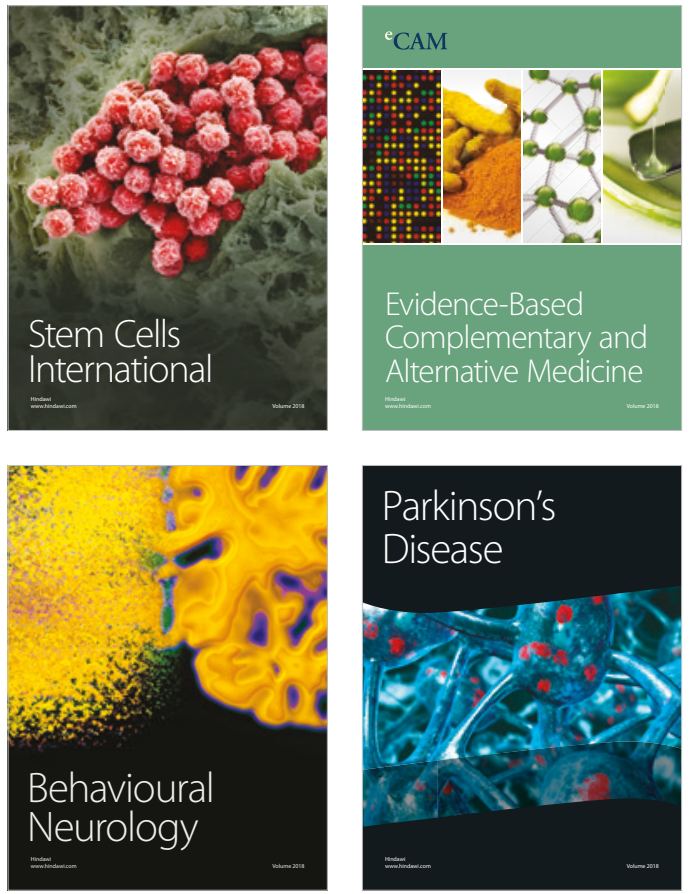

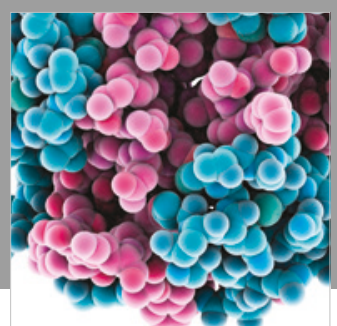

ournal of

Diabetes Research

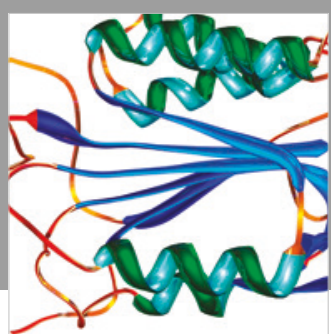

Disease Markers
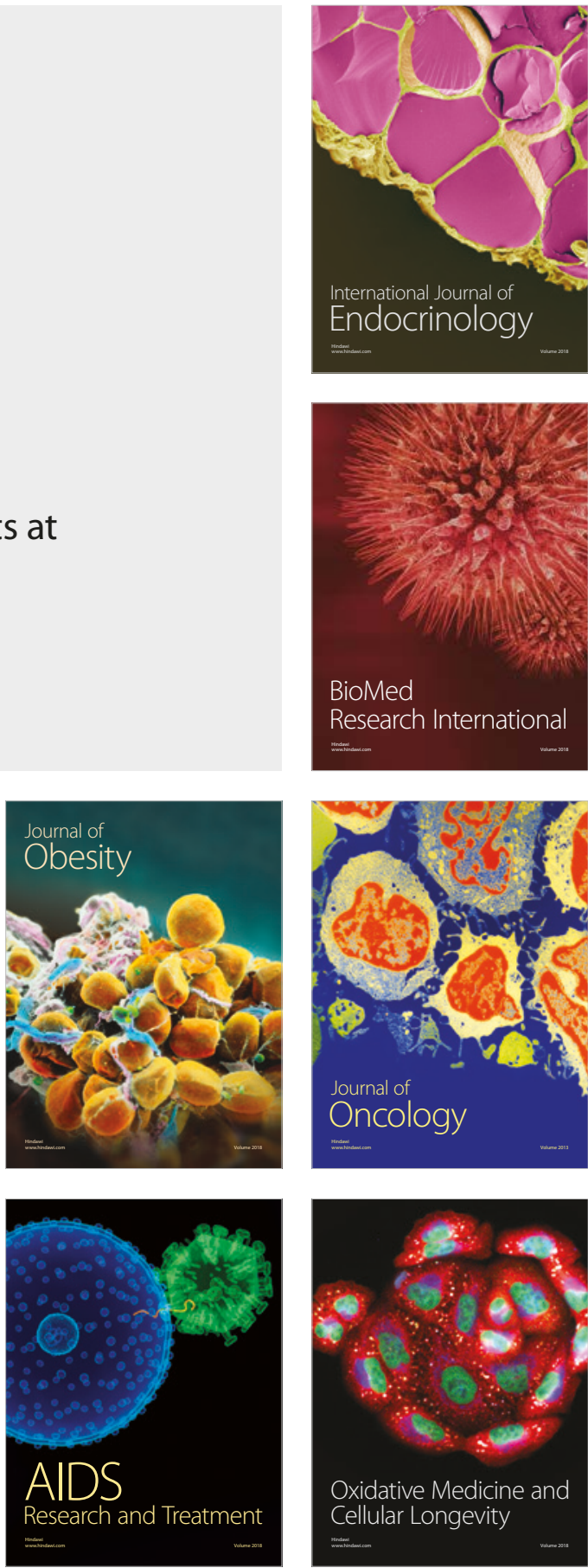